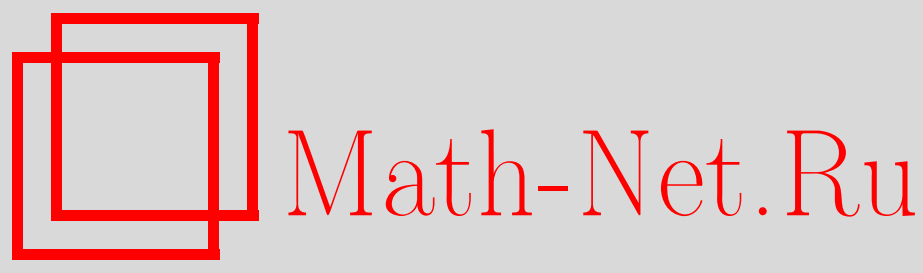

Н. Саикиа, С. А. С. Ахмед, Точное $S$-волновое решение уравнения Шредингера с помощью метода преобразования, ТМФ, 2011, том 168, номер 2, 291-298

DOI: https://doi.org/10.4213/tmf6682

Использование Общероссийского математического портала Math-Net.Ru подразумевает, что вы прочитали и согласны с пользовательским соглашением http://www.mathnet.ru/rus/agreement

Параметры загрузки:

IP : 54.198 .55 .26

26 апреля 2023 г., $17: 29: 11$

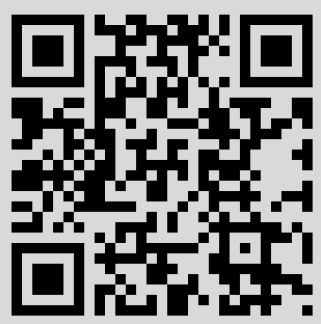




\title{
ТОЧНОЕ $S$-ВОЛНОВОЕ РЕШЕНИЕ УРАВНЕНИЯ ШРЕДИНГЕРА С ПОМОЩЬЮ МЕТОДА ПРЕОБРАЗОВАНИЯ
}

\begin{abstract}
Метод расширенных преобразований применен к потенциалу нестепенного вида для получения точно решаемых квантовых систем в пространствах любой размерности. Выведены точные аналитические решения уравнений Шредингера с точно решаемым потенциалом нестепенного вида. Для полученных в результате преобразования потенциалов рассчитаны квантованный спектр энергий связанных состояний и соответствующие волновые функции.
\end{abstract}

Ключевые слова: уравнение Шредингера, расширенное преобразование, точно решаемые потенциалы.

\section{1. ВВЕДЕНИЕ}

Потенциал, управляющий квантовой системой ( $\mathrm{KC})$, очень часто не позволяет получить точное решение уравнения Шредингера, что заставляет прибегать к различным приближенным схемам. С другой стороны, для достижения достаточной точности расчетов в рамках приближенных схем требуется, чтобы точно решаемый потенциал по возможности почти не отличался от заданного потенциала. Это заставляет нас искать столь много точно решаемых потенциалов, сколь возможно. В последнее время были предприняты значительные усилия для получения точного решения уравнения Шредингера с потенциалами, представляющими интерес с физической точки зрения [1]-[6]. Для расширения набора точно решаемых потенциалов мы развивали процедуру отображения [7]-[13], называемую методом расширенного преобразования (РП), в которое входит преобразование координат, последующее преобразование функций, а также набор подходящих подстановок. В настоящей работе мы применяем метод РП к точно решаемой $\mathrm{KC}$ из работы [12], чтобы генерировать новые $\mathrm{KC}$ в $D$-мерных пространствах; в каждой такой $\mathrm{KC}$ существуют нормированное точное аналитическое решение и соответствующие собственные значения энергии. В случае нестепенного потенциала метод РП можно применять много раз, выбирая различными способами слагаемые из многочленного потенциала,

*Department of Physics, Gauhati University, Guwahati, India.

E-mail: n_saikia06@yahoo.co.in 
чтобы получить разнообразные новые КС. Исключение составляет выбор слагаемых, который возвращает нас к исходной КС.

Работа организована следующим образом. В разделе 2 приведен формализм метода РП. В разделах 3, 4 описано применение РП к системе Морзе, в том числе показано, что́ получается в результате двукратного применения РП. В этих же разделах изложена процедура генерации КС типа системы Штурма и обсуждается метод перегруппировки параметров, переводящий зависящие от энергии $\mathrm{KC} \mathrm{в}$ нормальные/физические КС. В разделе 5 представлены обсуждения и выводы из наших исследований.

\section{2. ФОРМАЛИЗМ РП}

Рассмотрим радиальную часть уравнения Шредингера для исходной точно решаемой КС (системы А) с нестепенным центральным потенциалом, обозначаемым через $V_{A}(r)$, в $D_{A}$-мерном евклидовом пространстве $(\hbar=1,2 m=1)$ :

$$
\varphi_{A}^{\prime \prime}(r)+\frac{D_{A}-1}{r} \varphi_{A}^{\prime}(r)+\left[\epsilon_{n}^{A}-V_{A}(r)\right] \varphi_{A}(r)=0,
$$

где нормированная собственная функция $\varphi_{A}(r)$ и собственные значения $\epsilon_{n}^{A}$ для заданного потенциала $V_{A}(r)$ известны. Штрих обозначает дифференцирование волновой функции по ее аргументу.

Заменим переменные в уравнении (1) так, чтобы получающееся уравнение имело тот же вид, но содержало новые параметры. С этой целью применим РП [8], которое включает в себя преобразование координат $r \rightarrow g_{B}(r)$ и последующее преобразование функции

$$
\varphi_{B}(r)=\frac{\varphi_{A}\left(g_{B}(r)\right)}{f_{B}(r)},
$$

где $g_{B}(r)$ - функция преобразования, которая является непрерывной и, по крайней мере, трижды дифференцируемой, а $f_{B}(r)$ - зависящая от $r$ амплитуда, которая подлежит определению. Получаем следующее уравнение:

$$
\begin{aligned}
\varphi_{B}^{\prime \prime}(r)+ & \left(\frac{d}{d r} \ln \frac{f_{B}^{2}(r) g_{B}^{D_{A}-1}(r)}{g_{B}^{\prime}(r)}\right) \varphi_{B}^{\prime}(r)+\left(\frac{d}{d r} \ln f_{B}(r) \cdot \frac{d}{d r} \ln \frac{f_{B}^{\prime}(r) g_{B}^{D_{A}-1}(r)}{g_{B}^{\prime}(r)}+\right. \\
& \left.+g_{B}^{\prime 2}(r)\left[\epsilon_{n}^{A}-V_{A}\left(g_{B}(r)\right)\right]\right) \varphi_{B}(r)=0 .
\end{aligned}
$$

Данное дифференциальное уравнение второго порядка нужно преобразовать к виду, аналогичному уравнению Шредингера (1); размерность $D_{B}$ евклидова пространства преобразованной $\mathrm{KC}$ (системы $B$ ) теперь можно выбрать произвольной. Для этого потребуем, чтобы коэффициент при $\varphi_{B}^{\prime}(r)$ в уравнении $(3)$ удовлетворял равенству

$$
\frac{d}{d r} \ln \frac{f_{B}^{2}(r) g_{B}^{D_{A}-1}(r)}{g_{B}^{\prime}(r)}=\frac{D_{B}-1}{r},
$$

которое фиксирует $f_{B}$ как функцию от $g_{B}(r)$ и ее производной. Интегрирование этого уравнения дает

$$
\frac{1}{f_{B}(r)}=\frac{N_{B}}{\sqrt{g_{B}^{\prime}(r)}} \cdot g_{B}^{\left(D_{A}-1\right) / 2}(r) \cdot r^{-\left(D_{B}-1\right) / 2},
$$


где $N_{B}$ - нормировочная константа. Таким образом, из уравнения (2) с учетом (5) получаем

$$
\varphi_{B}(r)=\frac{N_{B}}{\sqrt{g_{B}^{\prime}(r)}} \cdot g_{B}^{\left(D_{A}-1\right) / 2}(r) \cdot r^{-\left(D_{B}-1\right) / 2} \cdot \varphi_{A}\left(g_{B}(r)\right) .
$$

Соответствующее $D_{B}$-мерное уравнение Шредингера для $\mathrm{KC} B$ можно записать как

$$
\begin{aligned}
\varphi_{B}^{\prime \prime}(r)+ & \frac{D_{B}-1}{r} \varphi_{B}^{\prime}(r)+\left(\frac{1}{2} \frac{g_{B}^{\prime \prime}(r)}{g_{B}^{\prime}(r)}+\frac{3}{4}\left(\frac{g_{B}^{\prime \prime}(r)}{g_{B}^{\prime}(r)}\right)^{2}+g_{B}^{\prime 2}(r)\left[\epsilon_{n}^{A}-V_{A}\left(g_{B}(r)\right)\right]-\right. \\
& \left.-\frac{D_{A}-1}{2} \frac{D_{A}-3}{2}\left(\frac{g_{B}^{\prime}(r)}{g_{B}(r)}\right)^{2}+\frac{D_{B}-1}{2} \frac{D_{B}-3}{2} \frac{1}{r^{2}}\right) \varphi_{B}(r)=0 .
\end{aligned}
$$

Из $V_{A}(r)$ выделяется одно или несколько слагаемых, тем самым задается так называемый рабочий потенциал, определяющий $g_{B}(r)$; мы будем обозначать его через $V_{A}^{(\mathrm{w})}\left(g_{B}(r)\right)$. Если потенциал $\mathrm{KC} A$ содержит $n$ слагаемых, то рабочий потенциал можно выбрать $2^{n}-1$ различными способами (напомним, выбор, возвращающий нас к $V_{A}\left(g_{B}(r)\right)$, мы исключаем), различные рабочие потенциалы приводят к различным функциям преобразования $g_{B}(r)$. Далее мы предполагаем, что рабочий потенциал содержит один член. РП преобразует (7) в дифференциальное уравнение второго порядка, которое можно привести к виду уравнения Шредингера. Для этого нужно воспользоваться следующими подходящими подстановками, которые по сути являются неотъемлемой частью метода преобразования:

$$
\begin{gathered}
g_{B}^{\prime 2}(r) V_{A}^{(\mathrm{w})}\left(g_{B}(r)\right)=-\epsilon_{n}^{B}, \\
g_{B}^{\prime 2}(r) \epsilon_{n}^{A}=-V_{B}^{(1)}(r), \\
g_{B}^{\prime 2}(r)\left[V_{A}\left(g_{B}(r)\right)-V_{A}^{(\mathrm{w})}\left(g_{B}(r)\right)\right]=V_{B}^{(2)}(r), \\
-\frac{1}{2}\left\{g_{B}(r), r\right\}+\frac{D_{A}-1}{2} \frac{D_{A}-3}{2}\left(\frac{g_{B}^{\prime}(r)}{g_{B}(r)}\right)^{2}-\frac{D_{B}-1}{2} \frac{D_{B}-3}{2} \frac{1}{r^{2}}=V_{B}^{(3)}(r),
\end{gathered}
$$

где $V_{B}^{(1)}(r)+V_{B}^{(2)}(r)+V_{B}^{(3)}(r)=V_{B}(r)$. После этого обычная форма уравнения Шредингера для КС $B$ принимает вид

$$
\varphi_{B}^{\prime \prime}(r)+\frac{D_{B}-1}{r} \varphi_{B}^{\prime}(r)+\left[\epsilon_{n}^{B}-V_{B}(r)\right] \varphi_{B}(r)=0 .
$$

В случае нестепенного потенциала, вообще говоря, нельзя убрать зависимость от $\epsilon_{n}^{B}$ из $V_{B}^{(1)}(r)$, в результате порождаемая $\mathrm{KC}$ оказывается системой типа Штурма (зависящей от энергии). Чтобы сделать ее нормальной/физической $\mathrm{KC}$, нам потребуются специальные приемы перегруппировки для каждого случая, коль скоро это возможно.

Важное свойство метода преобразования заключается в том, что волновые функции порождаемых $\mathrm{KC}$ почти всегда можно нормировать. Условие нормируемости для собственных функций $D_{B}$-мерной $\mathrm{KC} B$ имеет вид

$$
\int_{0}^{\infty} \varphi_{B}^{2}(r) r^{D_{B}-1} d r=\frac{1}{\left|N_{B}\right|^{2}}<\infty
$$


а нормировочная константа равна

$$
N_{B}=\left(\frac{-\epsilon_{n}^{B}}{\left\langle V_{A}^{(\mathrm{w})}\left(g_{B}(r)\right)\right\rangle}\right)^{1 / 2}
$$

Среднее значение точно решаемого потенциала $V_{A}\left(g_{B}(r)\right)$ всегда конечно, и поэтому среднее значение его части также конечно. Таким образом, нормируемы все функции $\varphi_{B}(r)$, для которых $\epsilon_{n}^{B} \neq 0$, если только $g_{B}(r)$ не ведет себя плохо. В той мере, в какой нас интересует ее поведение в нуле и на бесконечности, метод преобразования переносит свойство нормируемости исходной КС на дочернюю (порождаемую) КС.

\section{3. ГЕНЕРАЦИЯ ТОЧНОГО АНАЛИТИЧЕСКОГО РЕШЕНИЯ ИЗ ТОЧНО РЕШАЕМОГО НЕСТЕПЕННОГО ПОТЕНЦИАЛА}

В работе [12] мы рассмотрели центральный нестепенной потенциал, определяющий потенциал исходной $\mathrm{KC} A$ и точно решаемый только для случая $l=0(S$-волна), в трехмерном евклидовом пространстве. Этот потенциал имеет вид

$$
V_{A}(r)=4 \alpha^{2} \beta^{2} \operatorname{th}^{2}(\beta r)-\frac{\beta^{2}}{4} \operatorname{th}^{2}(\beta r)+\frac{3 \beta^{2}}{4} \operatorname{cosech}^{2}(\beta r),
$$

где $\alpha$ и $\beta$ - параметры потенциала. Собственные функции $S$-состояния записываются как

$$
\varphi_{A}(r)=\frac{N_{A}}{r} \frac{1}{\sqrt{\operatorname{th}(\beta r)}} \operatorname{sech}^{2(\alpha-n)}(\beta r){ }_{2} F_{1}\left(-n, 2 \alpha-n, 2 \alpha-2 n+1 ; \operatorname{sech}^{2}(\beta r)\right),
$$

где нормировочная константа равна

$$
N_{A}=\frac{2 \Gamma(2 \alpha-n)}{\Gamma(2 \alpha-2 n+1) \Gamma(n+1)} \sqrt{n(2 \alpha-n+1)(2 \alpha-n)} .
$$

Соответствующие собственные значения суть

$$
\epsilon_{n}^{A}=-\beta^{2}\left[4(\alpha-n)^{2}-\left(4 \alpha^{2}-\frac{1}{4}\right)\right] .
$$

Квантовые числа ограничены условием $\alpha>n+1 / 2$, и существует конечное число связанных состояний.

3.1. Первый порядок преобразования. Как было отмечено выше, КС $A$ допускает $2^{3}-1=7$ вариантов выбора рабочего потенциала, поскольку ее потенциал является трехчленным. Выбирая $V_{A}^{(\mathrm{w})}\left(g_{B}(r)\right)=-\left(\beta^{2} / 4\right) \operatorname{th}^{2}\left(\beta g_{B}(r)\right)$ в качестве рабочего потенциала, а также используя подстановку (8), находим функцию преобразования

$$
g_{B}(r)=\frac{1}{\beta} \operatorname{arch}\left(e^{\beta \xi_{n} r}\right), \quad \xi_{n}=\sqrt{\frac{4 \epsilon_{n}^{B}}{\beta^{2}}} .
$$

Постоянную интегрирования мы полагаем равной нулю, чтобы для $g_{B}(r)$ выполнялись требуемые условия $g_{B}(0)=0$ и $g_{B}(\infty)=\infty$. Функция преобразования $(16)$ вместе с равенством (9) дает

$$
V_{B}^{(1)}(r)=-C_{B}^{2} \frac{e^{2 \beta \xi_{n} r}}{2 \beta \xi_{n} r-1},
$$


где $C_{B}^{2}$ - характеристическая постоянная $\mathrm{KC} B$, полученная из преобразования КС $A$ и равная

$$
C_{B}^{2}=\frac{\xi_{n}^{2} \epsilon_{n}^{A}}{\beta^{2}}
$$

Из этого равенства мы получаем собственные значения для энергии $\mathrm{KC} B$ :

$$
\epsilon_{n}^{B}=-\frac{C_{B}^{2}}{4\left[4(\alpha-n)^{2}-\left(4 \alpha^{2}-1 / 4\right)\right]} .
$$

Уравнение (10) с учетом (16) дает

$$
\begin{aligned}
& V_{B}^{(2)}(r)=4 \alpha^{2} \beta^{2} \xi_{n}^{2}+\frac{3}{4} \beta^{2} \xi_{n}^{2} \frac{e^{2 \beta \xi_{n} r}}{\left(2 \beta \xi_{n} r-1\right)^{2}}, \\
& V_{B}^{(3)}(r)=\frac{1}{4} \frac{\beta^{2} \xi_{n}^{2}}{\left(2 \beta \xi_{n} r-1\right)^{2}}-\beta^{2} \xi_{n}^{2} \frac{e^{2 \beta \xi_{n} r}}{\left(2 \beta \xi_{n} r-1\right)^{2}}+\frac{D_{B}-1}{2} \frac{D_{B}-3}{2} \frac{1}{r^{2}} .
\end{aligned}
$$

Многочленный потенциал $\mathrm{KC} B$ имеет вид

$$
V_{B}(r)=4 \alpha^{2} \beta^{2} \xi_{n}^{2}-C_{B}^{2} \frac{e^{2 \beta \xi_{n} r}}{2 \beta \xi_{n} r-1}-\frac{1}{4} \frac{\beta^{2} \xi_{n}^{2}}{\left(2 \beta \xi_{n} r-1\right)^{2}}+\frac{D_{B}-1}{2} \frac{D_{B}-3}{2} \frac{1}{r^{2}} .
$$

Основная трудность, постоянно возникающая при работе с нестепенными потенциалами, заключается в том, что потенциал $V_{B}(r)$ всегда является потенциалом типа Штурма, который характеризуется зависимостью от $n$, таким образом, $n$ перестает быть квантовым числом и становится индексом, описывающим систему. КС $B$ типа Штурма можно преобразовать в нормальную/физическую КС с помощью специальных для каждого случая приемов перегруппировки, когда мы должны переопределить параметры $\mathrm{KC} A$. Чтобы сделать $\xi_{n}$ не зависящим от $n$, совершим замену параметра $\mathrm{KC} A \beta \rightarrow \beta_{n}$ так, что комбинация $s=\beta_{n} \xi_{n}$ перестанет зависеть от $n$; здесь мы ввели масштабный фактор $s$. Это приводит к тому, что потенциал $V_{B}(r)$ также перестает зависеть от $n$ и принимает вид

$$
V_{B}(r)=4 \alpha^{2} s^{2}-C_{B}^{2} \frac{e^{2 s r}}{2 s r-1}-\frac{1}{4} \frac{s^{2}}{(2 s r-1)^{2}}+\frac{D_{B}-1}{2} \frac{D_{B}-3}{2} \frac{1}{r^{2}} .
$$

Точная собственная функция порожденной $\mathrm{KC} B$ получается из уравнения (6):

$$
\varphi_{B}(r)=N_{B} r^{-\left(D_{B}-1\right) / 2} e^{-2(\alpha-n) s r}{ }_{2} F_{1}\left(-n, 2 \alpha-n, 2 \alpha-2 n+1 ; e^{-2 s r}\right) .
$$

3.2. Второй порядок преобразования. Применяя РП к $\mathrm{KC} B$, мы можем генерировать еще одну $\mathrm{KC}$, назовем ее $C$, задаваемую уравнением $(7)$, в котором нужно заменить индексы $B$ на $C$, а индексы $A$ - на $B$. Из многочленного потенциала $\mathrm{KC} B(22)$ для задания рабочего потенциала выбираем слагаемое

$$
-\frac{1}{4} \frac{\beta^{2} \xi_{n}^{2}}{\left(2 \beta \xi_{n} r-1\right)^{2}}
$$


откуда получаем

$$
\begin{gathered}
g_{C}^{\prime 2}(r)\left(-\frac{1}{4} \frac{\beta^{2} \xi_{n}^{2}}{\left(2 \beta \xi_{n} r-1\right)^{2}}\right)=-\epsilon_{n}^{C} \\
\frac{1}{2} \frac{g_{C}^{\prime \prime}(r)}{g_{C}^{\prime}(r)}+\frac{3}{4}\left(\frac{g_{C}^{\prime \prime}(r)}{g_{C}^{\prime}(r)}\right)^{2}+g_{C}^{\prime 2}(r)\left[\epsilon_{n}^{B}-V_{B}\left(g_{C}(r)\right)\right]- \\
-\frac{D_{B}-1}{2} \frac{D_{B}-3}{2}\left(\frac{g_{C}^{\prime}(r)}{g_{C}(r)}\right)^{2}+\frac{D_{C}-1}{2} \frac{D_{C}-3}{2} \frac{1}{r^{2}}=\epsilon_{n}^{C}-V_{C}(r) .
\end{gathered}
$$

Функцию преобразования находим с помощью интегрирования уравнения (26):

$$
g_{C}(r)=\frac{1}{\gamma} \ln \sec \left(\gamma \tau_{n} r\right), \quad \tau_{n}=\sqrt{\frac{4 \epsilon_{n}^{C}}{\gamma^{2}}},
$$

где теперь нам удобнее обозначить $\beta_{n} \xi_{n}$ через $\gamma$. Потенциал новой порожденной $\mathrm{KC} C$ равен $\left(D_{B}=3\right)$

$$
\begin{aligned}
V_{C}(r)= & 3 \gamma^{2} \tau_{n}^{2} \operatorname{cosec}^{2}\left(2 \gamma \tau_{n} r\right)-\tau_{n}^{2}\left(C_{B}^{2}+\gamma^{2}\right) \sec \left(\gamma \tau_{n} r\right)+ \\
& +\left(4 \alpha^{2} \gamma^{2} \tau_{n}^{2}-C_{C}^{2}\right) \operatorname{tg}^{2}\left(\gamma \tau_{n} r\right)-\frac{D_{C}-1}{2} \frac{D_{C}-3}{2} \frac{1}{r^{2}}
\end{aligned}
$$

Потенциал $V_{C}(r)$ зависит от $n$, т. е. является потенциалом типа Штурма, поскольку $\tau_{n}$ зависит от $n$. Чтобы привести потенциал к нормальному виду, используем ту же процедуру, что и для $\mathrm{KC} B$, когда мы преобразовывали параметр $\mathrm{KC} B$. Таким образом, получаем, что $\gamma_{n} \tau_{n}=a-$ масштабный фактор и

$$
\tau_{n}^{2} C_{B}^{2}=\tau_{n}^{2} \frac{\xi_{n}^{2} \epsilon_{n}^{A}}{\beta^{2}}=b^{2}
$$

Теперь КC $C$ характеризуется следующим не зависящим от $n$ потенциалом:

$$
\begin{aligned}
V_{C}(r)= & 3 a^{2} \operatorname{cosec}^{2}(2 a r)-\left(a^{2}+b^{2}\right) \sec ^{2}(a r)+ \\
& +\left(4 \alpha^{2} a^{2}-C_{C}^{2}\right) \operatorname{tg}^{2}(a r)-\frac{D_{C}-1}{2} \frac{D_{C}-3}{2} \frac{1}{r^{2}} .
\end{aligned}
$$

Собственные значения равны

$$
\epsilon_{n}^{C}=-\frac{a^{2} C_{C}^{2}}{C_{B}^{2}}\left[4(\alpha-n)^{2}-\left(4 \alpha^{2}-\frac{1}{4}\right)\right],
$$

где $\alpha>n$, таким образом, $V_{C}(r)$ порождает лишь конечное число связанных состояний. Соответствующие точные собственные функции $S$-волны для $\mathrm{KC} C$ можно получить из уравнения (6):

$$
\begin{aligned}
\varphi_{C}(r)= & N_{C} r^{-\left(D_{C}-1\right) / 2} \operatorname{ctg}^{2}(a r) \cos ^{2(\alpha-n)}(a r) \times \\
& \times{ }_{2} F_{1}\left(-n, 2 \alpha-n, 2 \alpha-2 n+1 ; \cos ^{2}(a r)\right) .
\end{aligned}
$$




\section{4. ДРУГОЙ ВЫБОР РАБОЧЕГО ПОТЕНЦИАЛА}

Выберем в качестве рабочего потенциала слагаемое

$$
\frac{3}{4} \operatorname{cosech}^{2}(\beta r)
$$

из многочленного потенциала (13) KC $A$. Выполним преобразование один раз. Используя подстановку (8), находим функцию преобразования

$$
g_{B}(r)=\frac{2}{\beta} \operatorname{arth}\left(e^{-\left(\beta \eta_{n} r+\phi\right)}\right), \quad \eta_{n}= \pm \frac{1}{\beta} \sqrt{-\frac{4 \epsilon_{n}^{B}}{3}} .
$$

Теперь нам необходимо найти энергию $\epsilon_{n}^{B} \mathrm{KC} B$. Чтобы удовлетворить граничные условия в нуле и на бесконечности для функции $g_{B}(r)$, необходимо выбрать знак минус перед квадратным корнем. Постоянная интегрирования - это постоянная фаза $\phi$, условие в нуле есть $g_{B}(0)=(2 / \beta) \operatorname{arth}\left(e^{\phi}\right) \neq 0$, а условие на бесконечности принимает вид $g_{B}(\infty)=(2 / \beta) \operatorname{arth}(0)=0$. Применяя эту функцию преобразования вместе с подстановками (9)-(11), для потенциала $\mathrm{KC} B$ получаем

$$
\begin{aligned}
V_{B}(r)= & -\frac{C_{B}^{2}+\eta_{n}^{2}}{4} \operatorname{cosech}^{2}\left(-\left(\eta_{n} r+\phi\right)\right)+\eta_{n}^{2}\left(16 \alpha^{2}-1\right) \operatorname{cosech}^{2}\left(-\left(2 \eta_{n} r+\phi\right)\right)+ \\
& +\frac{\eta_{n}^{2}}{4}-\frac{D_{B}-1}{2} \frac{D_{B}-3}{2} \frac{1}{r^{2}}
\end{aligned}
$$

Характеристическая постоянная $\mathrm{KC} B$ равна

$$
C_{B}^{2}=\frac{4 \eta_{n}^{2} \epsilon_{n}^{A}}{\beta^{2}} .
$$

Это “упрямый” потенциал типа Штурма, который невозможно преобразовать в нормальный/физический (не зависящий от $n$ ) потенциал. Выражение (36) используется для нахождения собственных значений энергии и соответствующих собственных функций:

$$
\begin{aligned}
\epsilon_{n}^{B}= & \frac{3 C_{B}^{2}}{4\left[16(\alpha-n)^{2}-\left(16 \alpha^{2}-1\right)\right]}, \quad \alpha>n, \\
\varphi_{B}(r)= & N_{B} r^{-\left(D_{B}-1\right) / 2} \operatorname{sh}^{1 / 2}\left(2 \eta_{n} r+\phi\right) \operatorname{th}^{2(\alpha-n)}\left(2 \eta_{n} r+\phi\right) \times \\
& \times{ }_{2} F_{1}\left(-n, 2 \alpha-n, 2 \alpha-2 n+1 ; \operatorname{th}^{2}\left(\eta_{n} r+\phi\right)\right) .
\end{aligned}
$$

Аналогично тому, как это было сделано выше, из уравнения (35), применяя РП к $\mathrm{KC} B$, можно генерировать $\mathrm{KC}$ второго поколения.

\section{5. ОБСУЖДЕНИЕ И ЗАКЛЮЧЕНИЕ}

Настоящая работа посвящена исследованию того, как порождаются новые вполне решаемые систем со связанными состояниями в нерелятивистском режиме путем преобразования в произвольном $D$-мерном пространстве. Для нахождения новых КС мы использовали метод РП. Для квантовых многочленных потенциалов можно генерировать конечное число различных точно решаемых $\mathrm{KC}$, выбирая различные 
рабочие потенциалы. Мы ограничились, однако, рабочим потенциалом, состоящим из одного члена. Рабочие потенциалы, состоящие из двух или многих членов, не рассматривались, поскольку их использование затруднено тем, что интеграл, задающий функцию преобразования $g(r)$, в большинстве случаев невозможно вычислить аналитически. Более того, если такие интегралы и можно найти, то они имеют вид $F(g(r))=r+C$, а обратную функцию $\left(F^{-1}(g)\right)(r)$ найти аналитически невозможно.

В настоящей работе для преобразования мы использовали точно решаемый нестепенной потенциал из работы [12]. Генерируемые потенциалы, вообще говоря, представляют собой потенциалы типа Штурма. Не существует общих процедур, которые можно было бы применить для преобразования их в нормальные потенциалы, однако имеется ряд примеров, когда можно применить специальные для каждого случая приемы, переопределить некоторые величины, и тем самым преобразовать КС типа Штурма в нормальные/физические КС. Если выбрать рабочий потенциал в виде $\left(-\beta^{2} / 4\right) \operatorname{th}^{2}(\beta r)$, то, применяя один раз РП, получаем $\mathrm{KC} B$ с потенциалом (23). Очевидно, что для генерации новых КС можно успешно применять РП сколько угодно раз, если мы рассматриваем нестепенные потенциалы. Однако важный момент, который необходимо отметить, заключается в том, что РП не имеют группового закона композиции, поскольку преобразование зависит от рабочего потенциала исходной КС. Поэтому множество РП, вообще говоря, не имеет групповой структуры. Обратное преобразование получается, если в качестве рабочего потенциала выбирается член, содержащий характеристическую постоянную, который получается из члена с энергией в исходной КС. Применяя второй раз РП с рабочим потенциалом, задаваемым выражением (25) (типа Штурма), к КC $B$, получаем $\mathrm{KC} \mathrm{с} \mathrm{потенциа-}$ лом (30) и конечным числом собственных состояний. Выбирая для задания рабочего потенциала слагаемое (33) в потенциале (13) $\mathrm{KC} A$, получаем "упрямый" потенциал (35) KC $B$ типа Штурма, который невозможно преобразовать к нормальному виду.

\section{Список литературы}

[1] P. M. Morse, Phys. Rev., 34:1 (1929), 57-64.

[2] N. Rosen, P. M. Morse, Phys. Rev., 42:2 (1932), 210-217.

[3] G. P. Flessas, Phys. Lett. A, 72:4-5 (1979), 289-290.

[4] F. Steiner, Phys. Lett. A, 106:8 (1984), 363-367.

[5] R. Dutt, A. Khare, U. P. Sukhatme, Amer. J. Phys., 56:2 (1988), 163-168.

[6] A. de Souza Dutra, Phys. Lett. A, 131:6 (1988), 319-321.

[7] S. A. S. Ahmed, Internat. J. Theoret. Phys., 36:8 (1977), 1893-1905.

[8] N. Saikia, S. A.S. Ahmed, Phys. Scr., 81:3 (2010), 035006, 6 pp.

[9] S. A. S. Ahmed, L. Buragohain, N. Saikia, Internat. J. Pure Appl. Phys., 4:3 (2008), $233-250$.

[10] N. Saikia, S. A. S. Ahmed, Latin.-Amer. J. Phys. Educ., 4:1 (2010), 61-66.

[11] S. A. S. Ahmed, L. Buragohain, Phys. Scr., 80:2 (2009), 025004, 6 pp.

[12] S. A.S. Ahmed, B. C. Borah, D. Sharma, Eur. Phys. J. D, 17:1 (2001), 5-11.

[13] S. A. S. Ahmed, L. Buragohain, Latin.-Amer. J. Phys. Educ., 3:3 (2009), 573-577. 